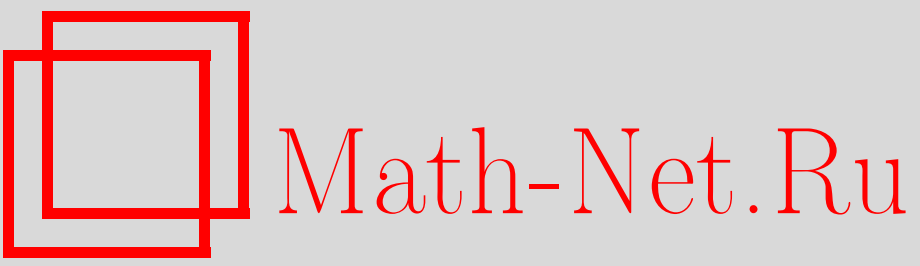

Р. Эрнандес Эредеро, Д. Леви, П. Винтерниц, Симметрии дискретного нелинейного уравнения Шредингера, ТМФ, 2001, том 127, номер 3, 379-387

DOI: https://doi.org/10.4213/tmf465

Использование Общероссийского математического портала Math-Net.Ru подразумевает, что вы прочитали и согласны с пользовательским соглашением

http://www . mathnet.ru/rus/agreement

Параметры загрузки:

IP : 54.224 .60 .19

26 апреля 2023 г., 02:45:29 
ТЕОРЕТИЧЕСКАЯ

И МАТЕМАТИЧЕСКАЯ

ФИЗИКА

Том 127, № 3

июнь, 2001

(C) 2001 г. $\quad$ Р. Эрнандес Эредеро* , Д. Леви ${ }^{\dagger}$, П. Винтерниц

\section{СИММЕТРИИ ДИСКРЕТНОГО НЕЛИНЕЙНОГО УРАВНЕНИЯ ШРЕДИНГЕРА}

Описаны алгебры Ли $L(h)$ точечных симметрий дискретного аналога нелинейного уравнения Шредингера (НУШ). В непрерывном пределе дискретное уравнение преобразуется в НУШ, а структура алгебры Ли меняется: происходит сжатие с шагом решетки $h$ в качестве параметра сжатия. Пятимерное подпространство в $L(h)$, порожденное как точечными, так и обобщенными симметриями, преобразуется в пятимерную алгебру точечных симметрий НУШ.

\section{1. ВВЕДЕНИЕ}

Мы исследуем алгебру Ли точечных симметрий и высшие симметрии иерархии дифференциально-разностных уравнений [1], связанных с дискретной спектральной задачей Захарова-Шабата

$$
\Phi_{n+1}=\left(Z+Q_{n} I_{1}+R_{n} I_{2}\right) \Phi_{n},
$$

где $\Phi_{n}=\Phi_{n}(z, t)-(2 \times 2)$-матричная волновая функция,

$$
Z=\left(\begin{array}{cc}
z & 0 \\
0 & \frac{1}{z}
\end{array}\right), \quad I_{1}=\left(\begin{array}{cc}
0 & 1 \\
0 & 0
\end{array}\right), \quad I_{2}=\left(\begin{array}{cc}
0 & 0 \\
1 & 0
\end{array}\right)
$$

а $z$ - собственное значение. Одним из членов иерархии является уравнение

$$
\dot{Q}_{n}=\frac{i \gamma}{h^{2}}\left[\left(1-\varepsilon\left|Q_{n}\right|^{2}\right)\left(Q_{n+1}+Q_{n-1}\right)-2 Q_{n}\right],
$$

которое мы назьваем дискретным нелинейнымм уравнением Шредингера (ДНУШ) и которое в непрерывном пределе $Q=h u, x=n h$ преобразуется в нелинейное уравнение Шредингера (НУШ)

$$
i u_{t}+\gamma u_{x x}-2 \varepsilon \gamma|u|^{2} u=0 .
$$

* Departamento de Física Teórica II, Facultad de Ciencias Físicas, Universidad Complutense de Madrid, Madrid, Spain. E-mail: rafahh@eucmos.sim.ucm.es

†Dipartimento di Fisica "Edoardo Amaldi", Universitá di Roma Tre and INFN, Roma, Italy. E-mail: levi@amaldi.fis.uniroma3.it

${ }^{\ddagger}$ Centre de Recherches Mathématiques, Université de Montréal, Montréal, Canada.

E-mail: wintern@CRM.UMontreal.CA 
Уравнение (2) является дифференциально-разностным уравнением с той же интегрируемой структурой, что и для НУШ. Его можно описать как интегрируемую дискретизацию НУШ или, точнее, как полудискретизацию, поскольку временна́я координата остается непрерывной. Говоря, что уравнение имеет “ту же интегрируемую структуру, что и НУШ", мы подразумеваем прежде всего, что оно допускает бесконечную иерархию коммутирующих потоков, которая в непрерывном пределе преобразуется в бесконечную иерархию потоков, коммутирующих с НУШ. Это связано с тем фактом, что соответствующие рекурсионные операторы порождают обе иерархии.

Настоящая работа является частью программы, направленной на использование теории интегрируемых систем для исследования лиевских симметрий дискретных уравнений. Рассматриваются разностные уравнения на регулярной решетке. Предыдушие исследования [2]-[6] показали, что классы точечных симметрий для разностных уравнений на регулярной решетке несколько ограничены. Многие интересные симметрии получаются только при одновременном рассмотрении групповых действий на конечном (или бесконечном) наборе точек решетки [7]-[9], а не просто в одной точке.

Основным результатом данной работы является анализ алгебры Ли симметрий ДНУШ (2) и ее соотношения с алгеброй Ли точечных преобразований НУШ (3). В частности, мы проверяем, что непрерывный предел из ДНУШ в НУШ соответствует сжатию алгебры Ли, как и в случае системы Тоды [2].

Сжатия алгебр Ли были впервые введены Инону и Вигнером [10] для изучения соотношения между релятивистскими и нерелятивистскими теориями при стремлении скорости света к бесконечности. Группа Лоренца тогда претерпевает "сжатие" к группе Галилея. В настоящее время существует обширная литература, касаюшаяся сжатий алгебр Ли (см. [11]-[13] и приведенную там литературу). В различных приложениях параметр сжатия оказьвался скоростью света, постоянной Планка, "радиусом" пространства положительной или отрицательной постоянной кривизны и др.

В нашем случае параметр сжатия является шагом решетки $h$. Бесконечномерная алгебра Ли $L(h)$ всех симметрий интегрируемого разностного уравнения сжимается к бесконечномерной алгебре $L(0)$ симметрий интегрируемого дифференциального уравнения. Основной момент состоит здесь в том, что конечномерное подмножество $S \subset L(h)$ сжимается к подалгебре в $L(0)$ - алгебре Ли $L_{p}$ точечных симметрий, тогда как само подмножество $S$ не образует алгебру Ли. Оно содержит как точечные, так и обобщенные симметрии дискретного уравнения и не замкнуто относительно коммутатора.

В разделе 2 мы используем рекурсионный оператор для вывода иерархии ДНУШ. В разделе 3 выписаны простейшие симметрии ДНУШ. Мы используем спектральное преобразование для связи изучаемых нелинейных эволюционных уравнений с линейными уравнениями для коэффициента отражения. Симметрии, действующие на пространстве решений эволюционного уравнения, преобразуются в симметрии, действуюшие в пространстве коэффициентов отражения, работать с которыми гораздо проше. Мы продолжаем вычисления коммутационных соотношений ранее найденных симметрий, выявляя структуру подмножества, которое мы используем. В разделе 4 описана предельная процедура, переводяшая ДНУШ в НУШ. Симметрии подвергаются той же предельной процедуре. Мы явным образом представляем непрерывные пределы тех симметрий, которые переходят в точечные симметрии, и показываем, что для получения всех точечных 
симметрий НУШ требуется включение обобщенных симметрий ДНУШ.

\section{2. ДИСКРЕТНЫЕ ИЕРАРХИИ НУШ}

Общая иерархия, которую мы изучаем, имеет вид [1]

$$
\left(\begin{array}{c}
\dot{R}_{n} \\
-\dot{Q}_{n}
\end{array}\right)+\omega(L)\left(\begin{array}{c}
R_{n} \\
Q_{n}
\end{array}\right)+\widetilde{\omega}(L)(2 n+1)\left(\begin{array}{c}
R_{n} \\
Q_{n}
\end{array}\right)=0,
$$

где $\omega(L)$ и $\widetilde{\omega}(L)$ - мероморфные функции рекурсионного оператора

$$
L\left(\begin{array}{c}
A_{n} \\
B_{n}
\end{array}\right)=\left(\begin{array}{c}
A_{n-1}-R_{n-1} a_{n} \sum_{j=n}^{\infty} \frac{Q_{j} A_{j}-R_{j} B_{j}}{1-R_{j} Q_{j}}-R_{n} \sum_{j=n}^{\infty}\left(Q_{j} A_{j-1}-R_{j} B_{j+1}\right) \\
B_{n+1}-Q_{n+1} a_{n} \sum_{j=n+1}^{\infty} \frac{Q_{j} A_{j}-R_{j} B_{j}}{1-R_{j} Q_{j}}-Q_{n} \sum_{j=n+1}^{\infty}\left(Q_{j} A_{j-1}-R_{j} B_{j+1}\right)
\end{array}\right)
$$

при $a_{n}=1-R_{n} Q_{n}$. Иерархия (4) связана с дискретной спектральной задачей (1). Когда потенциалы $R_{n}$ и $Q_{n}$ асимптотически ограничены, имеются корректно определенная спектральная задача и соответствуюшая обратная задача рассеяния. В этом случае уравнение (4) эквивалентно эволюции коэффициентов отражсения $\beta_{1}$ и $\beta_{2}$ :

$$
\left(\begin{array}{c}
\dot{\beta_{1}} \\
\dot{\beta}_{2}
\end{array}\right)+\omega(z)\left(\begin{array}{c}
\beta_{1} \\
\beta_{2}
\end{array}\right)+\widetilde{\omega}(z) z\left(\begin{array}{c}
\left(\beta_{1}\right)_{z} \\
\left(\beta_{2}\right)_{z}
\end{array}\right)=0
$$

Содержаший $\omega(z)$ член порождает изоспектральную эволющию, т.е. $\dot{z}=0$, тогда как член с $\widetilde{\omega}(z)$ приводит к неизоспектральной эволюции, т.е. $\dot{z}=\widetilde{\omega}(z)$.

Иерархию (4) можно редуцировать, полагая $R_{n}=\varepsilon Q_{n}^{*}$, что дает редуцированную иерархию

$$
\left(\begin{array}{c}
\varepsilon \dot{Q}_{n}^{*} \\
-\dot{Q}_{n}
\end{array}\right)+\left[\omega_{1}(L)-\omega_{1}^{*}\left(L^{-1}\right)\right]\left(\begin{array}{c}
\varepsilon Q_{n}^{*} \\
Q_{n}
\end{array}\right)+\left[\widetilde{\omega}_{1}(L)-\widetilde{\omega}_{1}^{*}\left(L^{-1}\right)\right](2 n+1)\left(\begin{array}{c}
\varepsilon Q_{n}^{*} \\
Q_{n}
\end{array}\right)=0
$$

где $\omega_{1}$ и $\widetilde{\omega}_{1}$ - аналитические функции, а звездочка обозначает комплексное сопряжение. Разлагая $\omega_{1}\left(z^{2}\right)=\alpha_{0}+\alpha_{1} z^{2}+\alpha_{2} z^{4}+\cdots$, получаем первые уравнения изоспектральной (т.е. независимой от $n$ ) иерархии

$$
\begin{aligned}
\dot{Q}_{n}= & \left(\alpha_{0}-\alpha_{0}^{*}\right) Q_{n}+\left(1-\varepsilon\left|Q_{n}\right|^{2}\right)\left(\alpha_{1} Q_{n+1}-\alpha_{1}^{*} Q_{n-1}\right)+\left(1-\varepsilon\left|Q_{n}\right|^{2}\right) \times \\
& \times\left\{\alpha_{2}\left[Q_{n+2}\left(1-\varepsilon\left|Q_{n+1}\right|^{2}\right)-\varepsilon Q_{n+1}\left(Q_{n}^{*} Q_{n+1}+Q_{n} Q_{n-1}^{*}\right)\right]-\right. \\
& \left.-\alpha_{2}^{*}\left[Q_{n-2}\left(1-\varepsilon\left|Q_{n-1}\right|^{2}\right)-\varepsilon Q_{n-1}\left(Q_{n}^{*} Q_{n-1}+Q_{n} Q_{n+1}^{*}\right)\right]\right\}+\cdots
\end{aligned}
$$

и неизоспектральной (т.е. зависящей от $n$ ) иерархии

$$
\begin{aligned}
\dot{Q}_{n}= & \left(\tilde{\alpha}_{0}-\tilde{\alpha}_{0}^{*}\right)(2 n+1) Q_{n}+\tilde{\alpha}_{1}\left[(2 n+3)\left(1-\varepsilon\left|Q_{n}\right|^{2}\right) Q_{n+1}+2 \varepsilon Q_{n} S_{n}^{*}\right]+ \\
& +\tilde{\alpha}_{1}^{*}\left[(2 n-1)\left(1-\varepsilon\left|Q_{n}\right|^{2}\right) Q_{n-1}+2 \varepsilon Q_{n} S_{n}\right]+\cdots
\end{aligned}
$$




\section{3. СИММЕТРИИ ДНУШ}

В общем случае $N$-е уравнения изоспектральной и неизоспектральной иерархий ДНУШ имеют вид

$$
\dot{Q}_{n}=\sum_{j=0}^{N}\left(\alpha_{j} B_{n}^{j}-\alpha_{j}^{*} \varepsilon A_{n}^{j}\right), \quad \dot{Q}_{n}=\sum_{j=0}^{N}\left(\tilde{\alpha}_{j} \widetilde{B}_{n}^{j}-\tilde{\alpha}_{j}^{*} \varepsilon \tilde{A}_{n}^{j}\right),
$$

где $A_{n}^{j}, B_{n}^{j}$ и $\tilde{A}_{n}^{j}, \widetilde{B}_{n}^{j}$ - вторые компоненты соответствующих выражений

$$
L^{-j}\left(\begin{array}{c}
\varepsilon Q_{n}^{*} \\
Q_{n}
\end{array}\right), \quad L^{j}\left(\begin{array}{c}
\varepsilon Q_{n}^{*} \\
Q_{n}
\end{array}\right), \quad L^{-j}\left[(2 n+1)\left(\begin{array}{c}
\varepsilon Q_{n}^{*} \\
Q_{n}
\end{array}\right)\right], \quad L^{j}\left[(2 n+1)\left(\begin{array}{c}
\varepsilon Q_{n}^{*} \\
Q_{n}
\end{array}\right)\right] .
$$

Пользуясь спектральным преобразованием, мы будем иметь дело с пространством коэффициентов отражения $\beta(z ; t)$. Иерархии $(7)$ преобразуются в линейные эволюции коэффициентов отражения:

$$
\beta_{t}+\sum_{j=0}^{N}\left(\alpha_{j} z^{2 j}-\alpha_{j}^{*} \varepsilon z^{-2 j}\right) \beta=0, \quad \beta_{t}+\sum_{j=0}^{N}\left(\tilde{\alpha}_{j} z^{2 j}-\tilde{\alpha}_{j}^{*} \varepsilon z^{-2 j}\right) z \beta_{z}=0 .
$$

Коммутируюшие потоки (симметрии), действуюшие в пространстве решений эволюционного уравнения, имеют аналоги в виде симметрий, действующих в пространстве коэффициентов отражения. Нас интересует ДНУШ (2), которое является изоспектральным уравнением для иерархий (7) при $N=1, \alpha_{0}=-i \gamma / h^{2}$ и $\alpha_{1}=i \gamma / h^{2}$. Чтобы найти достаточное число его симметрий, запишем анзац для эволюции коэффициента отражения

$$
\beta_{s}+\omega\left(z^{2}, t\right) \beta+\left(\gamma_{0}-\gamma_{0}^{*}+\gamma_{1} z^{2}-\gamma_{1}^{*} z^{-2}\right) z \beta_{z}=0 .
$$

Коммутируя эту эволюцию с соответствуюшей ДНУШ эволюцией (8), получаем

$$
\omega=\frac{2 i t \gamma}{h^{2}}\left[\left(\gamma_{0}-\gamma_{0}^{*}\right)\left(z^{2}-z^{-2}\right)+\gamma_{1}\left(z^{4}-1\right)-\gamma_{1}^{*}\left(1-z^{-4}\right)\right]+\widetilde{\omega}\left(z^{2}\right) .
$$

Эти симметрии можно записать как

$$
\begin{aligned}
\left(Q_{n}\right)_{s}= & \frac{2 i t \gamma}{h^{2}}\left[-\left(\gamma_{1}+\gamma_{1}^{*}\right) B_{0}+\left(\gamma_{0}-\gamma_{0}^{*}\right)\left(B_{1}-A_{1}\right)+\gamma_{1} B_{2}+\gamma_{1}^{*} A_{2}\right]+ \\
& +\left(\gamma_{0}-\gamma_{0}^{*}\right) \widetilde{B}_{0}+\gamma_{1} \widetilde{B}_{1}-\gamma_{1}^{*} \tilde{A}_{1}+\widetilde{\omega} .
\end{aligned}
$$

Таким образом, полагая $\widetilde{\omega}\left(z^{2}\right)=\omega_{0}+\omega_{1} z^{2}+\sigma_{1} z^{-2}+\omega_{2} z^{4}+\sigma_{2} z^{-4}$, получаем

$$
\begin{aligned}
\left(Q_{n}\right)_{s} & =\left(\gamma_{0}-\gamma_{0}^{*}\right)\left[\frac{2 i t \gamma}{h^{2}}\left(1-\varepsilon\left|Q_{n}\right|^{2}\right)\left(Q_{n+1}-Q_{n-1}\right)+(2 n+1) Q_{n}\right]+ \\
& +\gamma_{1}\left\{\frac{2 i t \gamma}{h^{2}}\left(1-\varepsilon\left|Q_{n}\right|^{2}\right)\left[Q_{n+2}-\varepsilon\left(Q_{n+1}^{*} Q_{n+2}+Q_{n}^{*} Q_{n+1}+Q_{n-1}^{*} Q_{n}\right) Q_{n+1}\right]-\right. \\
& \left.-\frac{2 i t \gamma}{h^{2}} Q_{n}+(2 n+3)\left(1-\varepsilon\left|Q_{n}\right|^{2}\right) Q_{n+1}+2 \varepsilon Q_{n} S_{n}^{*}\right\}+ \\
& +\gamma_{1}^{*}\left\{\frac{2 i t \gamma}{h^{2}}\left(1-\varepsilon\left|Q_{n}\right|^{2}\right)\left[Q_{n-2}-\varepsilon\left(Q_{n-1}^{*} Q_{n-2}+Q_{n}^{*} Q_{n-1}+Q_{n+1}^{*} Q_{n}\right) Q_{n-1}\right]-\right. \\
& \left.-\frac{2 i t \gamma}{h^{2}} Q_{n}-(2 n-1)\left(1-\varepsilon\left|Q_{n}\right|^{2}\right) Q_{n-1}-2 \varepsilon Q_{n} S_{n}\right\}+ \\
& +\omega_{0} Q_{n}+\omega_{1}\left(1-\varepsilon\left|Q_{n}\right|^{2}\right) Q_{n+1}+\sigma_{1}\left(1-\varepsilon\left|Q_{n}\right|^{2}\right) Q_{n-1}+ \\
& +\omega_{2}\left(1-\varepsilon\left|Q_{n}\right|^{2}\right)\left[Q_{n+2}-\varepsilon\left(Q_{n+1}^{*} Q_{n+2}+Q_{n}^{*} Q_{n+1}+Q_{n-1}^{*} Q_{n}\right) Q_{n+1}\right]+ \\
& +\sigma_{2}\left(1-\varepsilon\left|Q_{n}\right|^{2}\right)\left[Q_{n-2}-\varepsilon\left(Q_{n-1}^{*} Q_{n-2}+Q_{n}^{*} Q_{n-1}+Q_{n+1}^{*} Q_{n}\right) Q_{n-1}\right],
\end{aligned}
$$


а также следующие симметрии в векторной форме:

$$
\begin{aligned}
& X_{1}=Q_{n} \partial_{Q_{n}}-Q_{n}^{*} \partial_{Q_{n}^{*}}, \quad X_{2}=\left(1-\varepsilon\left|Q_{n}\right|^{2}\right) Q_{n+1} \partial_{Q_{n}}-\left(1-\varepsilon\left|Q_{n}\right|^{2}\right) Q_{n-1}^{*} \partial_{Q_{n}^{*}}, \\
& X_{3}=\left(1-\varepsilon\left|Q_{n}\right|^{2}\right) Q_{n-1} \partial_{Q_{n}}-\left(1-\varepsilon\left|Q_{n}\right|^{2}\right) Q_{n+1}^{*} \partial_{Q_{n}^{*}} \text {, } \\
& X_{4}=\left(1-\varepsilon\left|Q_{n}\right|^{2}\right)\left[Q_{n+2}\left(1-\varepsilon\left|Q_{n+1}\right|^{2}\right)-\varepsilon Q_{n+1}\left(Q_{n}^{*} Q_{n+1}+Q_{n-1}^{*} Q_{n}\right)\right] \partial_{Q_{n}}- \\
& -\left(1-\varepsilon\left|Q_{n}\right|^{2}\right)\left[Q_{n-2}^{*}\left(1-\varepsilon\left|Q_{n-1}\right|^{2}\right)-\varepsilon Q_{n-1}^{*}\left(Q_{n}^{*} Q_{n+1}+Q_{n-1}^{*} Q_{n}\right)\right] \partial_{Q_{n}^{*}}, \\
& X_{5}=\left(1-\varepsilon\left|Q_{n}\right|^{2}\right)\left[Q_{n-2}\left(1-\varepsilon\left|Q_{n-1}\right|^{2}\right)-\varepsilon Q_{n-1}\left(Q_{n}^{*} Q_{n-1}+Q_{n+1}^{*} Q_{n}\right)\right] \partial_{Q_{n}}- \\
& -\left(1-\varepsilon\left|Q_{n}\right|^{2}\right)\left[Q_{n+2}^{*}\left(1-\varepsilon\left|Q_{n+1}\right|^{2}\right)-\varepsilon Q_{n+1}^{*}\left(Q_{n} Q_{n+1}^{*}+Q_{n-1} Q_{n}^{*}\right)\right] \partial_{Q_{n}^{*}}, \\
& X_{6}=\left[-\frac{2 t \gamma}{h^{2}}\left(1-\varepsilon\left|Q_{n}\right|^{2}\right)\left(Q_{n+1}-Q_{n-1}\right)+(2 n+1) i Q_{n}\right] \partial_{Q_{n}}+ \\
& +\left[-\frac{2 t \gamma}{h^{2}}\left(1-\varepsilon\left|Q_{n}\right|^{2}\right)\left(Q_{n+1}^{*}-Q_{n-1}^{*}\right)-(2 n+1) i Q_{n}^{*}\right] \partial_{Q_{n}^{*}}, \\
& X_{7}=\left\{\frac { 2 i t \gamma } { h ^ { 2 } } ( 1 - \varepsilon | Q _ { n } | ^ { 2 } ) \left[Q_{n+2}-\varepsilon\left(Q_{n+1}^{*} Q_{n+2}+Q_{n}^{*} Q_{n+1}+Q_{n-1}^{*} Q_{n}\right) Q_{n+1}+\right.\right. \\
& \left.+Q_{n-2}-\varepsilon\left(Q_{n-1}^{*} Q_{n-2}+Q_{n}^{*} Q_{n-1}+Q_{n+1}^{*} Q_{n}\right) Q_{n-1}\right]- \\
& -\frac{4 i t \gamma}{h^{2}} Q_{n}+(2 n+3)\left(1-\varepsilon\left|Q_{n}\right|^{2}\right) Q_{n+1}+2 \varepsilon Q_{n} S_{n}^{*}- \\
& \left.-(2 n-1)\left(1-\varepsilon\left|Q_{n}\right|^{2}\right) Q_{n-1}-2 \varepsilon Q_{n} S_{n}\right\} \partial_{Q_{n}}+ \\
& +\left\{-\frac{2 i t \gamma}{h^{2}}\left(1-\varepsilon\left|Q_{n}\right|^{2}\right)\left[Q_{n-2}^{*}-\varepsilon\left(Q_{n-1} Q_{n-2}^{*}+Q_{n} Q_{n-1}^{*}+Q_{n+1} Q_{n}^{*}\right) Q_{n-1}^{*}+\right.\right. \\
& \left.+Q_{n+2}^{*}-\varepsilon\left(Q_{n+1} Q_{n+2}^{*}+Q_{n} Q_{n+1}^{*}+Q_{n-1} Q_{n}^{*}\right) Q_{n+1}^{*}\right]+ \\
& +\frac{4 i t \gamma}{h^{2}} Q_{n}^{*}-(2 n-1)\left(1-\varepsilon\left|Q_{n}\right|^{2}\right) Q_{n-1}^{*}-2 \varepsilon Q_{n}^{*} S_{n}^{*}+ \\
& \left.+(2 n+3)\left(1-\varepsilon\left|Q_{n}\right|^{2}\right) Q_{n+1}^{*}+2 \varepsilon Q_{n}^{*} S_{n}\right\} \partial_{Q_{n}^{*}}, \\
& X_{8}=\left\{-\frac{2 t \gamma}{h^{2}}\left(1-\varepsilon\left|Q_{n}\right|^{2}\right)\left[Q_{n+2}-\varepsilon\left(Q_{n+1}^{*} Q_{n+2}+Q_{n}^{*} Q_{n+1}+Q_{n-1}^{*} Q_{n}\right) Q_{n+1}-\right.\right. \\
& \left.-Q_{n-2}+\varepsilon\left(Q_{n-1}^{*} Q_{n-2}+Q_{n}^{*} Q_{n-1}+Q_{n+1}^{*} Q_{n}\right) Q_{n-1}\right]+ \\
& +(2 n+3) i\left(1-\varepsilon\left|Q_{n}\right|^{2}\right) Q_{n+1}+2 i \varepsilon Q_{n} S_{n}^{*}+ \\
& \left.+(2 n-1) i\left(1-\varepsilon\left|Q_{n}\right|^{2}\right) Q_{n-1}+2 i \varepsilon Q_{n} S_{n}\right\} \partial_{Q_{n}}+ \\
& +\left\{\frac { 2 t \gamma } { h ^ { 2 } } ( 1 - \varepsilon | Q _ { n } | ^ { 2 } ) \left[Q_{n-2}^{*}-\varepsilon\left(Q_{n-1} Q_{n-2}^{*}+Q_{n} Q_{n-1}^{*}+Q_{n+1} Q_{n}^{*}\right) Q_{n-1}^{*}-\right.\right. \\
& \left.-Q_{n+2}^{*}+\varepsilon\left(Q_{n+1} Q_{n+2}^{*}+Q_{n} Q_{n+1}^{*}+Q_{n-1} Q_{n}^{*}\right) Q_{n+1}^{*}\right]- \\
& -(2 n-1) i\left(1-\varepsilon\left|Q_{n}\right|^{2}\right) Q_{n-1}^{*}-2 i \varepsilon Q_{n}^{*} S_{n}^{*}- \\
& \left.-(2 n+3) i\left(1-\varepsilon\left|Q_{n}\right|^{2}\right) Q_{n+1}^{*}-2 i \varepsilon Q_{n}^{*} S_{n}\right\} \partial_{Q_{n}^{*}},
\end{aligned}
$$

где появляюшаяся в симметриях $X_{7}$ и $X_{8}$ функция $S_{n}$ определена как

$$
S_{n}=\sum_{j=n}^{\infty} Q_{j} Q_{j+1}^{*} .
$$


Если временна́я эволюция задается ДНУШ (2), можно охарактеризовать $S_{n}$ как функцию, удовлетворяющую системе

$$
\begin{gathered}
S_{n+1}=S_{n}-Q_{n} Q_{n+1}^{*} \\
\dot{S}_{n}=\frac{i \gamma}{h^{2}}\left[\left|Q_{n}\right|^{2}-\left(1-\varepsilon\left|Q_{n}\right|^{2}\right) Q_{n-1}^{*} Q_{n+1}\right] .
\end{gathered}
$$

Этим исчерпываются все симметрии ДНУШ, используемые в данной статье. Заметим, что точечными симметриями являются только $X_{1}, X_{2}, X_{3}$ и $X_{6}$, тогда как $X_{4}$ и $X_{5}$ обобшенные симметрии, а $X_{7}$ и $X_{8}$ - в высокой степени нелокальные симметрии.

Явно вычисленные коммутаторы симметрий представлены в табл. 1 , где символ $\gg$ обозначает симметрии высших порядков, которые не потребуются нам в дальнейших рассмотрениях.

Таблица 1

\begin{tabular}{|c|ccc|}
\hline & $X_{6}$ & $X_{7}$ & $X_{8}$ \\
\hline$X_{2}$ & $-2 i X_{2}$ & $-2 X_{4}+2 X_{1}$ & $-2 i X_{4}-2 i X_{1}$ \\
$X_{3}$ & $2 i X_{3}$ & $-2 X_{5}+2 X_{1}$ & $2 i X_{5}+2 i X_{1}$ \\
$X_{4}$ & $-4 i X_{4}$ & $\gg$ & $\gg$ \\
$X_{5}$ & $4 i X_{5}$ & $\gg$ & $\gg$ \\
$X_{6}$ & 0 & $2 X_{8}$ & $-2 X_{7}$ \\
$X_{7}$ & $-2 X_{8}$ & 0 & \\
\hline
\end{tabular}

Для вычисления коммутатора $\left[X_{7}, X_{8}\right]$ следует ввести дополнительные "нелокальности" (помимо $S_{n}$ ), нами здесь не рассматриваемые, так как получающаяся симметрия не сушественна в нашем исследовании.

Подалгебра, порожденная $X_{1}, X_{2}, X_{3}, X_{4}$ и $X_{5}$, является абелевой, и множество точечных симметрий является подалгеброй.

\section{4. НЕПРЕРЫВНЫЙ ПРЕДЕЛ}

Непрерывный предел

$$
Q=h u, \quad x=n h
$$

преобразует ДНУШ (2) в НУШ (3) с точностью до второго порядка,

$$
i u_{t}+\gamma u_{x x}-2 \varepsilon \gamma|u|^{2} u=O\left(h^{2}\right)
$$

Мы будем изучать соотношение между симметриями уравнения (2) и симметриями его непрерывного предела (3), особенно интересуясь нахождением прообразов точечных 
симметрий НУШ в алгебре симметрий уравнения (2).Нам потребуются следуюшие симметрии НУШ:

$$
\begin{aligned}
y_{1} \equiv & u \partial_{u}-u^{*} \partial_{u^{*}}, \quad y_{2} \equiv u_{x} \partial_{u}+u_{x}^{*} \partial_{u^{*}}, \quad y_{3} \equiv u_{t} \partial_{u}+u_{t}^{*} \partial_{u^{*}}, \\
y_{4} \equiv & \left(u_{x x x}-\varepsilon|u|^{2} u_{x}\right) \partial_{u}+\left(u_{x x x}^{*}-\varepsilon|u|^{2} u_{x}^{*}\right) \partial_{u^{*}}, \\
y_{5} \equiv & \left(u_{x x x x}-8 \varepsilon|u|^{2} u_{x x}-2 \varepsilon u^{2} u_{x x}^{*}-4 \varepsilon u\left|u_{x}\right|^{2}-6 \varepsilon u^{*} u_{x}^{2}+6|u|^{4} u\right) \partial_{u}- \\
& -\left(u_{x x x x}^{*}-8 \varepsilon|u|^{2} u_{x x}^{*}-2 \varepsilon u^{* 2} u_{x x}-4 \varepsilon u^{*}\left|u_{x}\right|^{2}-6 \varepsilon u u_{x}^{* 2}+6|u|^{4} u^{*}\right) \partial_{u^{*}}, \\
y_{6} \equiv & \left(i x u-2 \gamma t u_{x}\right) \partial_{u}-\left(i x u^{*}+2 \gamma t u_{x}^{*}\right) \partial_{u^{*}}, \\
y_{7} \equiv & \left(u+2 t u_{t}+x u_{x}\right) \partial_{u}+\left(u^{*}+2 t u_{t}^{*}+x u_{x}^{*}\right) \partial_{u^{*}}, \\
y_{8} \equiv & {\left[-2 \gamma^{2} t\left(u_{x x x}-6 \varepsilon|u|^{2} u_{x}\right)+x u_{t}+2 i \gamma u_{x}-2 i \gamma \varepsilon u \int|u|^{2} d x\right] \partial_{u}+} \\
& +\left[-2 \gamma^{2} t\left(u_{x x x}^{*}-6 \varepsilon|u|^{2} u_{x}^{*}\right)+x u_{t}^{*}-2 i \gamma u_{x}^{*}+2 i \gamma \varepsilon u^{*} \int|u|^{2} d x\right] \partial_{u^{*}} .
\end{aligned}
$$

Векторные поля $y_{1}, y_{2}, y_{3}, y_{6}$ и $y_{7}$ порождают подалгебру точечных симметрий, $y_{4}$ и $y_{5}$ являются обобшенными симметриями, а $y_{8}$ - нелокальными обобщенными симметриями, возникаюшими в нашем анализе. Замечательно, что подалгебра точечных симметрий НУШ пятимерна, тогда как подалгебра точечных симметрий ДНУШ (2) четырехмерна. Одна независимая точечная симметрия НУШ должна воспроизводиться из непрерывного предела некоторой обобщенной симметрии уравнения (2).

Непрерывный предел вспомогательной функции равен

$$
S \rightarrow-h \int|u|^{2} d x+h^{2} \int\left(u_{x} u^{*}-u u_{x}^{*}\right) d x+O\left(h^{3}\right) .
$$

Поэтому пределы подходяших комбинаций симметрий (т.е. таких, что их непрерывный предел дает обсуждавшееся выше независимое множество лиевских симметрий НУШ) имеют вид

$$
\begin{aligned}
& \bar{y}_{1} \equiv X_{1} \rightarrow y_{1}, \quad \bar{y}_{2} \equiv \frac{X_{2}-X_{1}}{h} \rightarrow y_{2}+O(h), \\
& \bar{y}_{3} \equiv \frac{i \gamma}{h^{2}}\left(X_{3}-2 X_{1}+X_{2}\right) \rightarrow y_{3}+O\left(h^{2}\right), \quad \bar{y}_{4} \equiv \frac{X_{4}+3 X_{1}-3 X_{2}-X_{3}}{h^{3}} \rightarrow y_{4}+O(h), \\
& \bar{y}_{5} \equiv \frac{X_{5}+6 X_{1}-4 X_{2}-4 X_{3}+X_{4}}{h^{4}} \rightarrow y_{5}+O(h), \\
& \bar{y}_{6} \equiv h \frac{X_{6}-i X_{1}}{2} \rightarrow y_{6}+O\left(h^{2}\right), \quad \bar{y}_{7} \equiv \frac{X_{7}}{4} \rightarrow y_{7}+O(h), \\
& \bar{y}_{8} \equiv \frac{\gamma}{2 h}\left(X_{8}-2 X_{6}-2 i X_{3}-2 i X_{2}+4 i X_{1}\right) \rightarrow y_{8}+O(h) .
\end{aligned}
$$

Все точечные симметрии ДНУШ в непрерывном пределе дают точечные симметрии НУШ. Но имеется одна дополнительная независимая точечная симметрия НУШ $\left(y_{7}\right)$, которая должна получаться из неточечных симметрий $\left(X_{7}\right)$ ДНУШ. Таким образом, происходит сжатие алгебры Ли симметрий ДНУШ.

2 Теоретическая и математическая физика, т. 127, № 3, 2001 г. 
В табл. 2 приведены коммутаторы для симметрий уравнения (2).

Таблица 2

\begin{tabular}{|l|ccc|}
\hline & $\bar{y}_{6}$ & $\bar{y}_{7}$ & $\bar{y}_{8}$ \\
\hline $\bar{y}_{2}$ & $-i \bar{y}_{1}-h i \bar{y}_{2}$ & $-\bar{y}_{2}+\frac{h i}{2 \gamma} \bar{y}_{3}-\frac{h^{2}}{2} \bar{y}_{4}$ & $-\bar{y}_{3}-h i \gamma \bar{y}_{4}$ \\
$\bar{y}_{3}$ & $2 \gamma \bar{y}_{2}+h i \bar{y}_{3}$ & $-2 \bar{y}_{3}-\frac{h^{2}}{2} i \gamma \bar{y}_{5}$ & $2 \gamma^{2} \bar{y}_{4}-h \gamma^{2} \bar{y}_{5}$ \\
$\bar{y}_{4}$ & $-\frac{3}{\gamma} \bar{y}_{3}-2 h i \bar{y}_{4}$ & $\gg$ & $\gg$ \\
$\bar{y}_{5}$ & $-4 i \bar{y}_{4}+2 h i \bar{y}_{5}$ & $\gg$ & $\gg$ \\
$\bar{y}_{6}$ & 0 & $\bar{y}_{6}+\frac{h}{2} i \bar{y}_{1}+\frac{h^{2}}{2 \gamma} \bar{y}_{8}-\frac{h^{3}}{2 \gamma} \bar{y}_{3}$ & $-2 \gamma \bar{y}_{7}+2 h \gamma \bar{y}_{2}+h^{2} i \bar{y}_{3}$ \\
$\bar{y}_{7}$ & $-\left[\bar{y}_{6}, \bar{y}_{7}\right]$ & 0 & \\
\hline
\end{tabular}

Таблица 3

\begin{tabular}{|c|ccc|}
\hline & $y_{6}$ & $y_{7}$ & $y_{8}$ \\
\hline$y_{2}$ & $-i y_{1}$ & $-y_{2}$ & $-y_{3}$ \\
$y_{3}$ & $2 \gamma y_{2}$ & $-2 y_{3}$ & $2 \gamma^{2} y_{4}$ \\
$y_{4}$ & $-3 / \gamma y_{3}$ & $-3 y_{4}$ & $-3 i \gamma y_{5}$ \\
$y_{5}$ & $-4 i y_{4}$ & $-4 y_{5}$ & $\gg$ \\
$y_{6}$ & 0 & $y_{6}$ & $-2 \gamma y_{7}$ \\
$y_{7}$ & $-y_{6}$ & 0 & $y_{8}$ \\
\hline
\end{tabular}

Из табл. 3, в которой представлены коммутаторы симметрий НУШ, можно видеть, как векторные поля $y_{1}, y_{2}, y_{3}, y_{6}$ и $y_{7}$ порождают подалгебру точечных симметрий уравнения (3), которая является образом порожденного элементами $\bar{y}_{1}, \bar{y}_{2}, \bar{y}_{3}, \bar{y}_{6}$ и $\bar{y}_{7}$ множества при сжатии. Множество, порожденное элементами $\bar{y}_{1}, \bar{y}_{2}, \bar{y}_{3}, \bar{y}_{6}$ и $\bar{y}_{7}$, содержит подалгебру точечных алгебр уравнения (2), но само не является алгеброй.

\section{5. ЗАКЛЮЧЕНИЕ}

Перечислим несколько обших выводов нашего исследования. Как уже отмечалось, если мы изучаем симметрии разностных уравнений на фиксированной и непреобразуемой решетке и хотим получить все симметрии, сушествующие в непрерывном пределе, то следует рассматривать обобщенные симметрии наряду с точечными. Линейные средства теории интегрируемых систем позволяют получить алгебру Ли симметрий рассматриваемого дискретного интегрируемого уравнения. Эта алгебра включает очень малую подалгебру точечных преобразований. В непрерывном пределе, при стремлении шагового параметра $h$ к нулю, структура алгебры Ли меняется. После сжатия $h \rightarrow 0$ эта алгебра по-прежнему является бесконечномерной и по-прежнему включает 
как обобщенные, так и точечные симметрии. Однако множество элементов алгебры симметрий дискретного уравнения, включающее как точечные, так и обобщенные симметрии, сжимается в чисто точечные симметрии соответствуюшего дифференциального уравнения. Происходят сжатия бесконечномерных алгебр Ли.

Возникает вопрос о том, как найти и использовать симметрии неинтегрируемых разностных уравнений, у которых нет линейной системы. Одна из возможностей состоит в том, чтобы отказаться от идеи фиксированных решеток, т.е. изучать группы преобразований, действуюших одновременно на уравнения и на решетку. Например, дилатации координат изменяют масштаб решетки. Такой подход был использован Дородницыным с соавторами [14], [15].

Другим подходом является предложенньй ранее метод дифференциальных уравнений [4]. Дифференциально-разностное уравнение рассматривается как бесконечный набор дифференциальных уравнений и ишутся точечные симметрии этой бесконечной системы. Таким образом, получаются некоторые, хотя и не обязательно все, обобщенные симметрии изучаемого дифференциально-разностного уравнения. Проблема состоит в том, что часто трудно решить соответствующий бесконечный набор определяющих уравнений.

Наконец, упомянем внутренний метод [4], который наиболее прост и дает точечные симметрии дискретного уравнения (на фиксированной решетке).

Благодарности. Работа была частично поддержана DGCYT (грант PB98-0821), HATO (грант 0717/96) и Universidad Complutense/del Amo Foundation, а также (П. В.) NSERC of Canada и FCAR du Québec.

\section{Список литературы}

[1] S. C. Chiu, J. F. Ladik. J. Math. Phys. 1977. V. 18. P. 690-700.

[2] R. H. Hernández Heredero, D. Levi, M. A. Rodríguez, P. Winternitz. J. Phys. A. 2000. V. 33. P. 5025-5040.

[3] D. Levi, M. A. Rodríguez. J. Phys. A. 1992. V. 25. P. L975-L979.

[4] D. Levi, P. Winternitz. Phys. Lett. A. 1991. V. 152. P. 335-338; J. Math. Phys. 1993. V. 34. P. 3713-3730.

[5] S. Maeda. IMA J. Appl. Math. 1987. V. 38. P. 129-134; Math. Japonica. 1980. V. 25. P. 405-420.

[6] G. R. W. Quispel, H.W. Capel, R. Sahadevan. Phys. Lett. A. 1992. V. 170. P. 379-383.

[7] R. Floreanini, L. Vinet. J. Math. Phys. 1995. V. 36. P. 7024-7042.

[8] R. H. Hernández Heredero, D. Levi, P. Winternitz. J. Phys. A. 1999. V. 32. P. 2685-2695.

[9] D. Levi, L. Vinet, P. Winternitz. J. Phys. A. 1997. V. 30. P. 633-649.

[10] E. Inönü, E. P. Wigner. Proc. Nat. Acad. Sci. USA. 1953. V. 39. P. 510-524.

[11] A. A. Izmest'ev, G.S. Pogosyan, A. N. Sissakian, P. Winternitz. J. Math. Phys. 1999. V. 40. P. 1549-1573.

[12] R. V. Moody, J. Patera. J. Phys. A. 1991. V. 24. P. 2227-2258.

[13] J. Patera, G. S. Pogosyan, P. Winternitz. J. Phys. A. 1999. V. 32. P. 805-826.

[14] В. А. Дородницын. Докл. РАН. 1993. Т. 328. № 6. С. 678-682.

[15] V. A. Dorodnitsyn, R. Kozlov, P. Winternitz. J. Math. Phys. 2000. V. 41. P. 480-504. 